\title{
Cognitive Radio Networking Model Using Intelligent Receptors Based Rewrite Techniques
}

\author{
Chandrasekaran Subramaniam, Arjun M., Sarathy Venkatesh T. J., and Veejay Adeesh
}

\begin{abstract}
The objective of the research work is to propose a cognitive radio networking model using intelligent receptors (IR) based rewrite techniques. The set of special function receptors in the cognitive engine within the radio framework senses the status of the bombarding channels and collects all the relevant details within the system and the outside environment. The proposed IR technique declares and defines a specific control path among number of choices meeting the requirements of the spectrum users and allocator. A formal model for cognitive radio with extended adaptability and control action has been proposed. The computational rule rewriting techniques to decide the best reaction to be taken by the cognitive engine among the prevailing issues like spectrum sharing, leasing and identification of hidden nodes and peak spectrum are used. The work improvises the performance, reliability and cost factors of the software defined network in a cognitive radio framework through receptor modules.
\end{abstract}

Index Terms-Reactive design, adaptability, cognition, rewrite rules, formal model, intelligent receptors, spectrum users.

\section{INTRODUCTION}

Cognitive Radio (CR) is a programmed and configured intelligent radio that can automatically detect available channels in a wireless spectrum and change transmission parameters. CR has the ability and capacity to support the end users and the network nodes. The task is to make the cognitive radio as a smart radio using intelligent receptors that can sense the external environment, learn from the history and make intelligent decisions to adjust to its transmission parameters according to the current state of the environment with or without attack. The capability of a CR is to learn, sense, measure and be aware of the parameters related to the channel characteristics, availability of spectrum, power, interference and noise temperature, radios operating environment, user requirements, infrastructure and nodes, local policies. Sensing poses a major challenge for the CRs as it has to be carried out without compromising on the performance and quality of the nodes.

Cognitive Radios may also impose various challenges due to ever increasing complexity of network architecture [1].

Manuscript received December 2, 2014; revised March 17, 2016.

Chandrasekaran Subramaniam is with the Easa College of Engineering and Technology, Coimbatore, Anna University, Chennai, India (e-mail: chandrasekaran_s@msn.in).

Arjun M. is with the Coimbatore Institute of Engineering and Technology, Coimbatore, Anna University, Chennai, India (e-mail: arjunmane@outlook.com).

Sarathy Venkatesh T. J. and Veejay Adeesh are with Karpagam College of Engineering, Anna University, Chennai, India (e-mail sarthytj@outlook.com, veejayadeesh614@gmail.com).
These CRs pose challenges at all levels of abstraction dealing with their RF and analogue design issues. This kind of radio technology allows the unlicensed operation to be in licensed band and also as a sensing environment and as a resource management environment [2]. The two techniques that could be used for spectrum sensing: "energy detection" and "feature detection". It is suggested that RF assisted spectrum sensing by block down conversion can increase probability of finding available channels [3]. A CR node constantly senses the spectrum to detect any changes in unused channels in the allocated spectrum. A frequency agile radio module is capable of reconfiguring RF and switching to newly selected frequency bands. A CR allows a single radio to provide a wide variety of functions acting as a cell phone, broadcast, sender, GPS receiver, wireless data terminal or node etc. A system level design methodology for mapping CR onto an MPSoC platform is also addressed in the earlier works by using a design methodology based on a task transaction level interface to partition the application into communicating tasks. In a bio inspired design approach, three bio-inspired approaches were discussed for distribution and sharing processes in CR networks [4]. The high cost of configuring and managing large-scale networks, the fluctuating nature of the available spectrum, diverse QoS requirements of various applications are to be solved. The intensifying difficulties of centralized control through channel management, cooperative spectrum sensing, bio-inspired spectrum sharing and network scalability are universally applicable to heterogeneous CRs [5]. The challenges that remain unaddressed are heterogeneity of CRs, spectrum management and sharing, Mutli-channel spectrum sensing, self control and self management is reqiured towards reliable CR's with high scalability and spectrum sensing speed. In the work by Saeed Alrabee et al., the problems addressed are network and application layer ssues like denial of Service, jamming, dyeing, malicious user attack. This work is to propose and formally specify the important ability of CRs using a set of bio inspired receptors like interfaces. These categories of like software defined receptors engaged by a set of rewrite rules can be used to realize the $\mathrm{CR}$ model with expected computational intelligence.

The organization of the paper is as follows: Section II explains the proposed intelligent receptor model in needed for the interactive design of cognitive radios with an architectural design through the workflow. Section III proposes a set of rewrite rules that can be executed to sense and monitor the spectrum towards channel management by the set of intelligent receptors to make the SDR and CR as adaptable. Section IV explains the reactive design aspects of the $\mathrm{CR}$ with rewrite rules towards a best case design in a fault free 
condition, minimum fault cases, a worst case with faulty processes and an all parallel design patterns of all receptors with simulation code. Section V concludes the work by a packet status of a CR with and without node faults along with the limitations and future work on CR design.

\section{Proposed Intelligent ReCEPtors Model For COGNITIVE RADIOS}

The proposed intelligent receptor model brings out a bio inspired approach of using a set of sensors or receptors like devices shown in Fig. 1. A programmable logic device with standard networking stacks is selected for the purpose of deploying software defined hardware components.

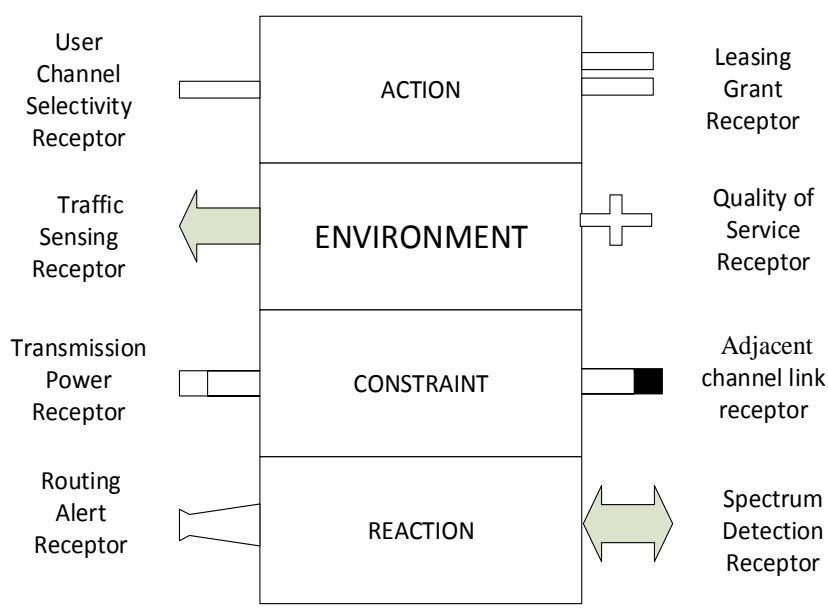

Fig. 1. Proposed architecture of CR-SDR with intelligent receptors.

These devices are continuously running a sample application which accepts any form of interrupt to capture the signal. This particular antenna like device controlled by an application that identifies the parameters or features of the event occurred around it. These are highly sensitive to electromagnetic signal and selective in capturing the frequency of the variations. The core modules are a conventional software defined radio and cognitive radio with rules embedded as shown in Fig. 2. The device is interacting with the outside environment using a set of input-output bidirectional lines. The receiving antenna defined by an application software captures these parameters through spectrum detection receptors (SDReceptor) for an intelligent detection of the surroundings. At the same time, a receptor like hardware antenna device receives the actual signal and also tuned to the nearby frequency in the spectrumThe application developed for these set of adjacent channel detector (ACReceptor). The image signal frequency may be calculated from the central frequency and the maximum deviation permitted for the nearby channel if any as per the digital communication regulation as per Equation 1.

$$
F_{a c}=F c \pm \Delta f
$$

If the spectrum is free and the channel bandwidth is within the available band, then that part of the spectrum is allowed and granted to that channel at that point of time. This is achieved by the software application called leasing grant receptor (LGReceptor) module within the programmable device. The programmable device has to sense the traffic around the receptors with the help of the data link layer modules through the traffic sensing receptor (TSReceptor). These receptors are software defined interfaces with a separate set of registers and counters. Once these registers and counters are filled with their maximum values, these receiving units can set suitable flags indicating that the traffic around the center frequency is maximum and raise interrupts to the core of SDR. The next set of activities are to determine the parameters of the much wanted channels and the arguments passed along with a function declaration. That is more number of functions are called to verify the bandwidth, latency and throughput of the channel for a sample of packets to know the quality of service of transmission in that specific channel by quality of service receptor like electromagnetic interferences. (QSReceptor) as shown in Fig. 2.

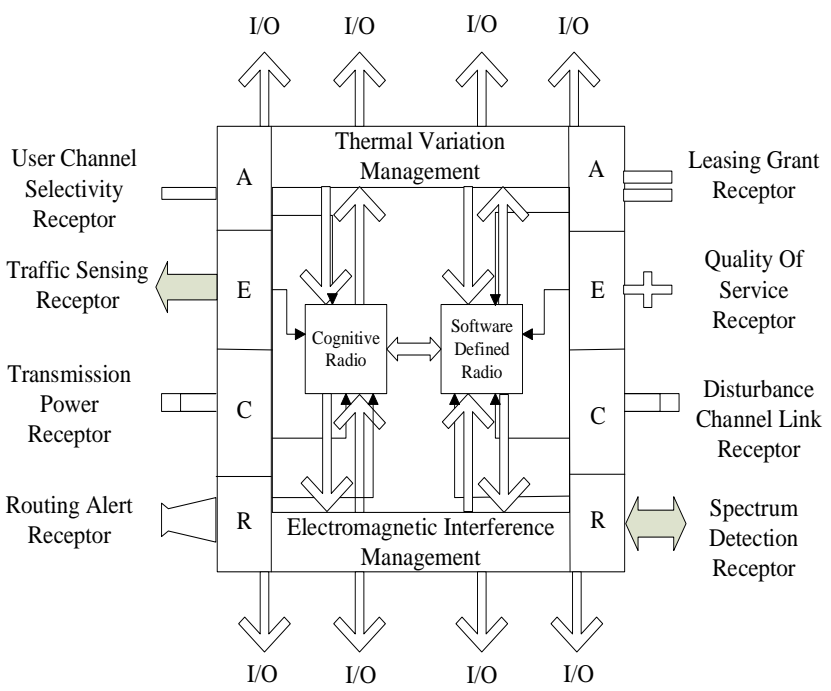

A-Action E-Environment C- Constraint R-Reaction Fig. 2. Channel action reaction model of receptors.

Once all these software defined functions are activated by the core SDR module, the most important umbrella activities are the power and thermal management functions are initiated concurrently. The remaining three set of receptors are assigned for the above management purpose of input and output device management. These goals are achieved by a set of transmission power receptor (TPReceptor), routing alert receptor (RAReceptor) and user channel selectivity receptor (USReceptor). These receptors are operating as per the rewrite rules embedded in the core of cognitive radio segments that will interact with the SDR and other peripheral devices. The Cognition Module for the $\mathrm{CR}$ where the cognition can be considered as a set of processes of acquiring knowledge and understanding through experience and senses. These processes include aspects such as bringing awareness, perception, reasoning and judgment. To automate these activities in a parallel manner, a set of software modules called receptors are designed and developed as shown in Fig. 2. The software modules consist of a bundle of rules that are to be rewritten whenever the underlying core requested them.

A sample rewrite rule (RR) set is given below: The sense rule by the SDReceptor can rewrite the following rule towards spectrum detection as the RR1, $R R 1 \rightarrow$ condition (bandwidth low) $\rightarrow$ channel utilization $\rightarrow$ Spectrum access 
The rule rewrite RR1 depicts the rule that governs the specified channel utilization when the transmission or reception procedure tries to access the spectrum that has to be rewritten when the system demands. Similarly the application developed for these set of adjacent channel detector (ACReceptor) will decide the sharing of same channel and permit other signals in the same spectrum. This can be written as a RR2.

\section{$R R 2 \rightarrow$ sharing and subleasing}

Many such rules are to rewritten by the receptors into the environment or into the corresponding subsystem modules. The last three rules are inculcating the cognition or system policies whenever the system wants to adapt according to the signal strength, noise, resources, environment or resetting when alert signal comes. RR3 can be depicted as

\section{$R R 3 \rightarrow$ Peaking support for traffic}

A number of actions such as securing the systems, optimizing the cost, verifying the license and taking care of privacy are done so as to ensure the proper functioning of the entire system. These policies are substantially taken care of by QSReceptor. This can be written as RR4.

\section{$R R 4 \rightarrow$ Policy (security, privacy, licensing, cost)}

Reconfiguration is applied when certain subsystem modules are deprived of refinement that is written as RR5.

\section{$R R 5 \rightarrow$ Smart antenna reconfiguration}

The software module is physically loaded from its own host memory or a virtual instance of other node's application is running can be represented by RR6 as shown below.

\section{$R R 6 \rightarrow$ Physical or virtual entity}

All these rules need not be rewritten in a static or preordered sequence. The individual receptors can pass their parameters after they have sensed and interacted with the modules where they have been embedded in the programmable hardware device. The type and the number of instances of the needed receptors are derived or instantiated from the respective receptor classes. The environment class is responsible for sensing the environment object which can be defined and declared as a meta-class. The system operates as a mixed hardware and software components which can be realized only when a workflow is defined in order to serialize the events and thus activities like a series- parallel components connected within a RF system. The typical workflow of activities performed by the receptors is depicted in Fig. 3 which is a compartmentalized parallel system executing the rules and rewriting the same rules whenever they are demanded. Each rectangular compartment is a container where all the rules inside of the container will be executed in parallel. The outer rectangle or container is the proposed system container as a whole. Once the rules inside each and every container has been rewritten, then the outside rules will be executed as P- system defined by Gabriel Ciobanu, Gheorghe Paun and Mario J. Perez-Jimenez as in a membrane computing system [6].

The actions into channel and reactions from the environment are carried out in a parallel manner in order to plan, decide and act accordingly. The rewrite theory automata rules follow the phases of cognition in the order of plan, decide and act.

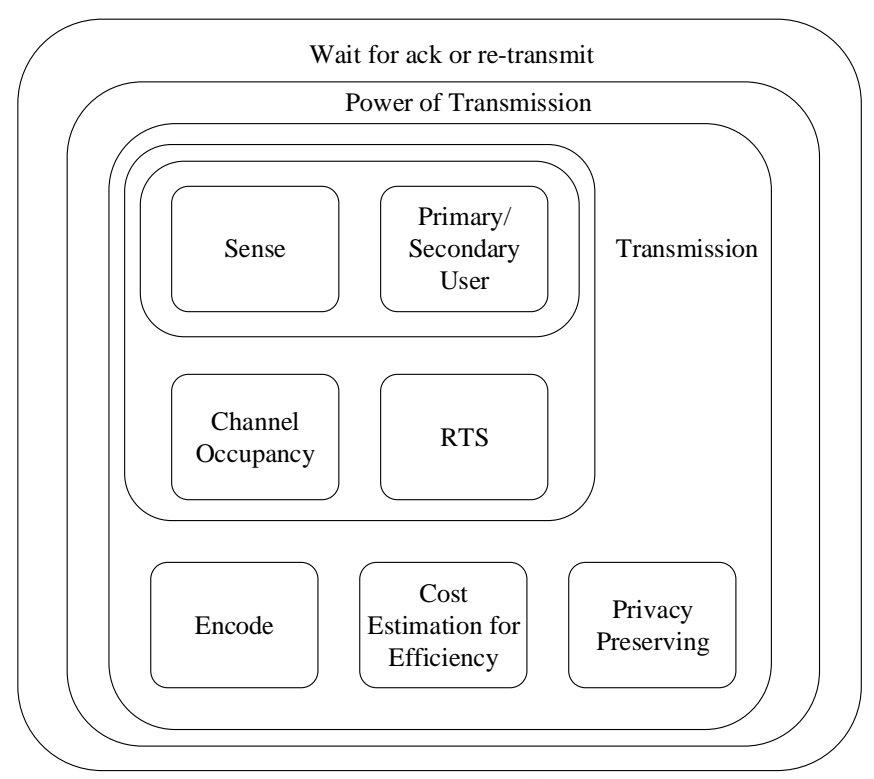

Fig. 3. Typical workflow.

\section{REWRITE RULES FOR RECEPTORS}

The rules or grammar for the CR-SDR-IR can be summarized for the effective and efficient operation of a radio system using software defined rule based modules. All these rules are to be written again and again whenever and wherever they are applicable.

In a case, wherever the user wants to check for the amount of radio interference (inf) in the allotted channel (ch) within the bandwidth (bd), the user(us) has to transmit a signal may be called as "Request to Send"(RTS). This rule can be represented as,

1) us bd ch inf $\rightarrow$ RTS

Once the detection receptor grants the access (ac) to the requested channel or bandwidth for transmission (tr) of a signal namely Clear to Send (CTS). This rule can be represented as,

2) R1(bd ac) (ch tr) $\rightarrow C T S$

The possible set of rules that are to be rewritten in all occasions through the receptors within the system.

Once the Power of Transmission (potr) reaches its maximum (mx) value, the receptor 4 re-routes (ro) the signal. This rule can be expressed as,

3) R4 potr $m x \rightarrow$ ro

When the channel (ch) utilization (ut) is maximum (mx), the Receptor 5 checks (ck) license (lic) for valid users. This rule can be expressed as,

4) $R 5$ (mx tr) (ch ut) $\rightarrow$ ck lic

Receptor 8 reconfigures (rcf) the transmitting antenna (an) when either transmission (se) or reception (re) is facing difficulties. This rule can be expressed as,

5) $R 8($ an !se) $\|(a n ! r e) \rightarrow r c f$

Receptor 8 reconfigures (rcf) the transmitting antenna (an) when there is difficulty in transmission (se). This rule can be expressed as,

6) $R 8$ (an!se) (an re) $\rightarrow r c f$ 
Receptor 8 reconfigures (rcf) the transmitting (se) antenna when reception (re) is facing difficulties. This rule can be expressed as,

7) $R 8$ (an se) (an!re) $\rightarrow r c f$

Reconfiguration is not required when the transmitting antenna (an) is not facing difficulties. This rule can be expressed as,

8) $R 8$ (an se) (an re) $\rightarrow r c f$

Minimal interference (inf) and data (da) loss (ls) represent the perfect flow of operation. This rule can be expressed as, 9) $R 5(! \operatorname{Inf})(d a ! l s) \rightarrow p f$

The Receptor 6 raises alert when the signal attenuation (sia), time delay (td) and interference (int) is below the desired level. This rule can be expressed as,

10) $R 6($ mx sia) $\|(m x$ tmd $) \|($ Int $m x) \rightarrow$ raisealert

The receptor 5 amplifies (amp) the signal when the signal intensity (In) is lower than the threshold (trshld) value. This rule can be expressed as,

\section{1) $R 5$ (In) $($ sig $<$ trshld $) \rightarrow a m p$}

The Receptor 8 allocates (allc) the available bandwidth (bd) for transmission (tr) among primary or secondary users (usr). This rule can be expressed as,

12) $R 8$ (allc bd) (allc tr) $\rightarrow$ usr

The Receptor 5 checks (ck) the primary user's (usr) license (lic) for validity (vld). This authentication could also be carried out using helper nodes [7], [8]. This rule can be expressed as,

\section{3) $R 5$ (ck lic) (usr) $\rightarrow$ vld}

The Receptor 7 routes (ro) the signal (sg) based on the available bandwidths (bd). This rule can be expressed as,

14) $R 7$ ro sg $\rightarrow$ bd

Signal is re transmitted (rtr) when the intensity (In) of the signal to be sent, is low (lw). This rule can be expressed as,

15) (lw In) $\rightarrow$ rtr

Skips a node (skipnode) if the transmission (tr) initialization exceeds the pre-defined time-out (tout) value. This rule can be expressed as,

16) (!tr) (tout) $\rightarrow$ skipnode

A mutual exclusion (mex) is provided to the primary (pm) users in the network to ensure their utilizing (ut) space isn't interfered. This rule can be expressed as,

17) (pm.sg) (ut) $\rightarrow$ mex

This mutual exclusion (mex) is removed when the primary (pm) user's utilization (ut) of the licensed channel remains idle. This provides a path for the secondary (sg) users to access the idle spectrum. This rule can be expressed as,

18) (pm.sg) (!ut) $\rightarrow$ !mex

Secondary (sg) users utilize (ut) the idle spectrum. This rule can be expressed as,

19) !mex $\rightarrow$ (sg) (ut)

Receptor 5 acknowledges (ack) the signals with lower interference (inf). This rule can be expressed as,

20) R5 (! inf) (sig) $\rightarrow$ ack

\section{REACTIVE DESIGN IN CRN}

The reactive design of a $\mathrm{CR}$ with intelligent receptors can be explained with the help of multiple graphs towards the behavior of CR. This is indicated the various actions, constraints and the reaction to or from the environment in different scenarios.

\section{Variables: Frequency, Power, Channel, Constants: Wait, Sense, Act, React, Halt}

Wait activity is represented as a vertical line segment "Wait |"(W). Sensing activity is represented by a horizontal line segment of the same size, "Sense ---" (S). Any kind of action as per the rule is represented by a right inclined line segment, "Act / " (A). And the last reaction activity is represented by a left inclined line segment as, "React $\backslash(\mathbf{R})$ ".

The visual representation of various scenarios and the corresponding receptors actions and reactions as per the rules can be given by a tree like structure. Each and every activity is carried out as rewriting the rules at different points of time in different circumstances. The tree like structure thus obtained will be the same for all three variables considered. There are three important scenarios with which the reactive design in the intelligent behavior of the receptors can be explored. Fig. 4(a) denotes the best possible case where there are minimum actions and fault free conditions.
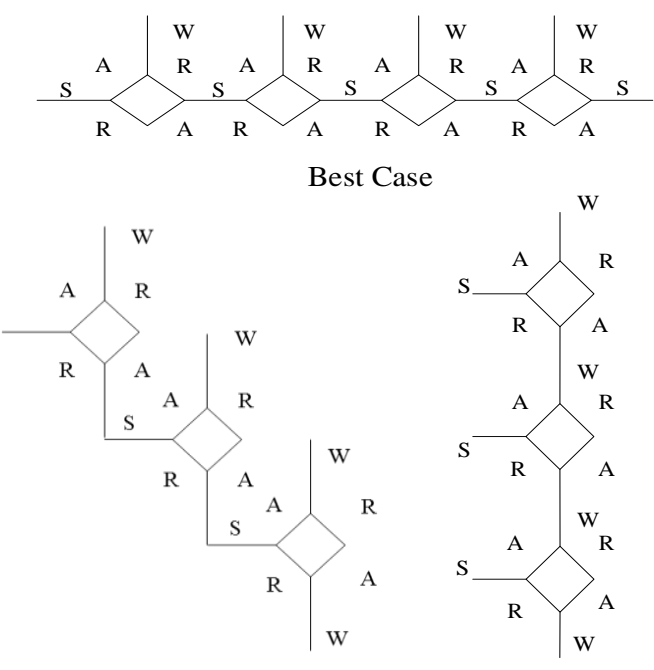

Minimum Fault Case

Worst Case

Sense -> (act | react) $\rightarrow$ (react |act) -> sense

Fig. 4. (a) Best case; (b) Minimal Fault case; (c) Worst cases.

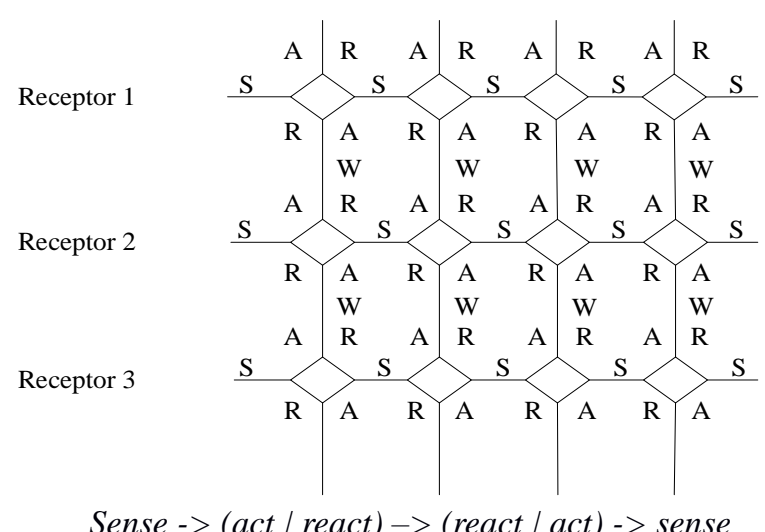

Fig. 5. Parallel actions of all receptors.

The best possible case in the behavior of CR and the worst case with non-favorable conditions from the system or environment are expressed in terms of system variables and local variables at that instant of time as shown below: The 
receptor takes the straight path when the rules are satisfied. Fig. 4(b) denotes that if a particular receptor gets damaged while performing an action, then it backtracks to the previous action such that it can start anew to achieve its goal.

This case of receptors responding where maximum number of wait stages, sense, act, react tasks are to be carried out leading to a maximum delay in the cognition process. That is if any receptor gets damaged in the long run, then they would backtrack to their previous state and take an alternative path.

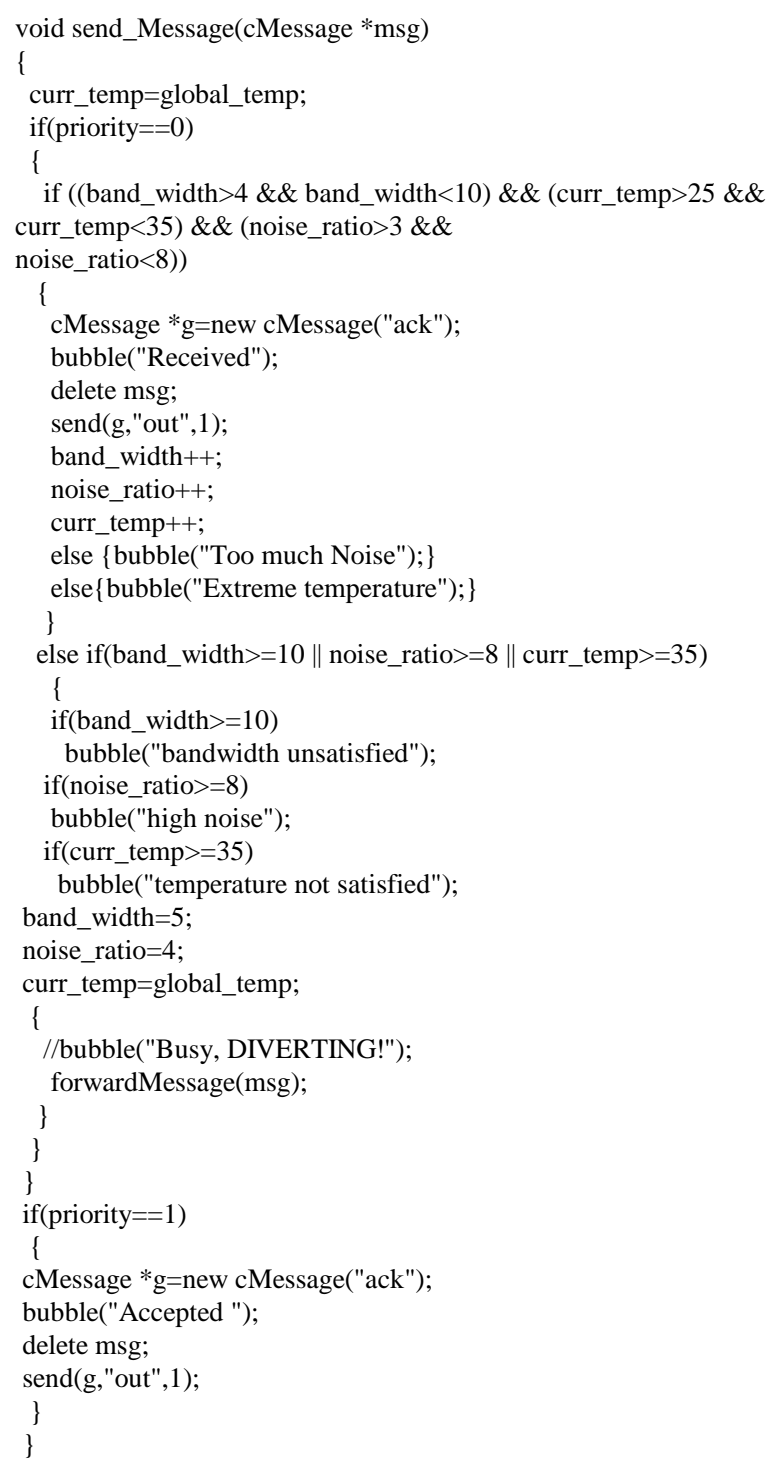

Fig. 4(c) denotes that the receptors senses the environment simultaneously in their own respects and acts accordingly. Here, every receptor fails to achieve its goal and again tries to sense for new nodes in the environment. Because of this repetitive failures, the design takes more time to complete all the functions as worst case scenario shown. Similarly the receptors function according to their rules. This system could be eventually developed as the mesh network shown in Fig. 5. The parallel action of multiple receptors as and when triggered respond to the requests of the input or output signals and the membrane like environment [7]. All phases of wait, sense, act, and react are performed when all receptors are collaborating with each other to perform the radio communication tasks. The simulation tool that has been used is OMNet++. The specific topology, transmission and reception is achieved using a Network Description file (.NED).

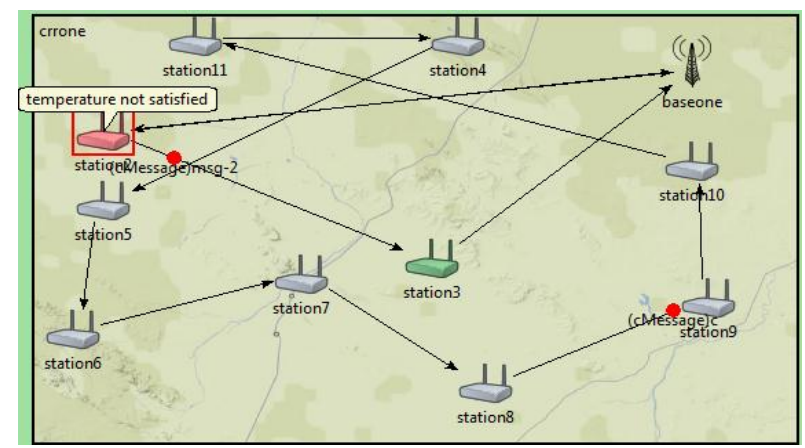

Fig. 7. Constraint not satisfied.

The message handling functionality of the nodes are provided through a $\mathrm{C}++$ source file, part is shown in Fig. 6 . The nodes encapsulate various receptors which in turn are based on pre-defined set of logical rules. In order to allow a message to be transmitted to another node, all the constraints have to be met as shown in Fig. 7 .

TABLE I: CHANNEL ACTION REACTION APPROACH

\begin{tabular}{|c|c|c|}
\hline Channel & Action & Reaction \\
\hline Free & $\begin{array}{l}\text { Sense the Channel } \\
\text { Occupancy }\end{array}$ & $\begin{array}{l}\text { Pool RTS from willing } \\
\text { nodes. }\end{array}$ \\
\hline Congested & Sense Node Overhead & $\begin{array}{l}\text { Temporarily suspend } \\
\text { nodes with lower priority }\end{array}$ \\
\hline Interference & $\begin{array}{l}\text { Sense for damaged } \\
\text { data at the receiving } \\
\text { end. }\end{array}$ & $\begin{array}{l}\text { Redirect channel path } \\
\text { Re transmit } \\
\text { unacknowledged data }\end{array}$ \\
\hline Occupied & $\begin{array}{l}\text { Sense the Channel } \\
\text { Occupancy }\end{array}$ & Wait till time out. \\
\hline Temperature & Sense the environment & The packet is diverted \\
\hline
\end{tabular}

The Action Reaction (Table I) specifies rational actions that are carried out to get to know the status of the channels. When the status of a channel is known to the node, some actions can be taken by the receptors. These possible actions are given under the 'reaction' tab. The reactions are derived through the set of rules. Here, all possible states of the available channels that could be utilized, are considered (Table II).

TABLE II: PACKET STATUS SUMMARY

\begin{tabular}{|c|c|c|c|}
\hline Source & Destination & Rules inferred & Status \\
\hline $\begin{array}{l}\text { Baseon } \\
\text { e }\end{array}$ & $\begin{array}{l}\text { Station } 2(\mathrm{P} \\
\mathrm{M})\end{array}$ & $\begin{array}{l}\text { The message 'msg- } 1 \text { ' is } \\
\text { transmitted }\end{array}$ & Accepted \\
\hline $\begin{array}{l}\text { Station } \\
2(\mathrm{PM})\end{array}$ & Baseone & $\begin{array}{l}\text { The message is } \\
\text { acknowledged }\end{array}$ & \\
\hline $\begin{array}{l}\text { Baseon } \\
\mathrm{e}\end{array}$ & $\begin{array}{l}\text { Station2(P } \\
\text { M) }\end{array}$ & $\begin{array}{l}\text { The message ' } m s g-2 \text { ' is } \\
\text { transmitted }\end{array}$ & Accepted \\
\hline $\begin{array}{l}\text { Station } \\
2(\mathrm{PM})\end{array}$ & Baseone & $\begin{array}{l}\text { The message is } \\
\text { acknowledged }\end{array}$ & \\
\hline $\begin{array}{l}\text { Baseon } \\
\mathrm{e}\end{array}$ & $\begin{array}{l}\text { Station2(P } \\
\text { M) }\end{array}$ & $\begin{array}{l}\text { Station } 2 \text { becomes inactive. } \\
\text { Packet is diverted }\end{array}$ & Diverted \\
\hline $\begin{array}{l}\text { Station } \\
2(\mathrm{PM})\end{array}$ & $\begin{array}{l}\text { Station3(S } \\
\text { M) }\end{array}$ & $\begin{array}{l}\text { Inactive primary user acts as a } \\
\text { passive router to } \\
\text { accommodate the secondary } \\
\text { user for the time being. }\end{array}$ & Accepted \\
\hline $\begin{array}{l}\text { Station } \\
3(\mathrm{SM})\end{array}$ & Baseone & $\begin{array}{l}\text { Station } 3 \text { acknowledges radio } \\
\text { packet. }\end{array}$ & $\begin{array}{l}\text { Channel } \\
1\end{array}$ \\
\hline
\end{tabular}




\section{CONCLUSION}

The reactive design of a cognitive radio and its network using the proposed set of intelligent receptor model is achieved using the rule rewrite techniques. The set of software defined modules carry out the rewrite process in the cognitive engine connected with a SDR and IO. The rules are derived to incorporate the sensing the environment, channel allocation, traffic management and monitoring the working or operation frequency and simulate. The rules are derived and executed on the simulator with the code to validate the performance of the application. The limitation of the proposed model is the determination of minimum number of frequency domain Level Crossing Rate with the detailed specifications like Channel Impulse response (CIR) estimation and the variation of channel estimates can also be carried in the same intelligent receptor model in the future work.

\section{REFERENCES}

[1] W. El-Hajj, H. Safa, and M. Guizani, "Survey of security issues in cognitive radio networks," Journal of Internet Technology, vol. 12, no. 2, pp. 1-7, 2011.

[2] S. Alrabaee, A. Agarwal, N. Goel, M. Zaman, and M. Khasawneh, "Higher layer issues in cognitive radio network," in Proc. ICACCI, 2012, pp. 325-329.

[3] B. Razavi, "Cognitive radio design challenges and techniques," IEEE Journal of Solid-State Circuits, vol. 45, no. 8, pp 1542-1551, August 2010.

[4] Y. T. Hou, A. M. Wyglinski, M. Nekovee, H. G. Zhang, R. Chandramouli, and F. Martin, Guest Editorial: Special Issue on Cognitive Radio Oriented Wireless Networks ANS Communications, Published Online, Springer, June 18, 2008, pp. 412-413.

[5] Z. S. Zhang and K. P. Long, "Self-organization paradigms and optimization approaches for cognitive radio technologies: A survey," IEEE Wireless Communications, pp. 36-42, April 2013.

[6] G. Ciobanu, G. Paun, and M. J. Perez-Jimenez, Applications of Membrane Computing, Springer-Verlag Berlin Heidelberg, 2006, pp. 253-293.

[7] H. Arslan, Cognitive Radio, Software Defined Radio, and Adaptive Wireless Systems, Springer, pp. 235-259.
[8] Y. Liu, P. Ning, and H. Dai "Authnticating primary users signals in cognitive radio networks via integerated cryptographic and wireless link signatures," in Proc. 2010 IEEE Symposium on Security and Privacy, pp. 286-301.

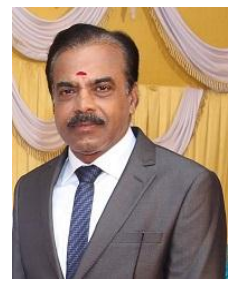

Chandrasekaran Subramiam was born at Uttukuli, Tirupur Dist., India. He has thirty-five years of acaddemic and research experience, completed and awarded under graduation, graduation and research Ph.D. degrees from Anna University, Chennai.

He has published more than 100 international publications in various international conferences and journals.

Prof. Chandrasekaran has been a member of IEEE, ACM, WSEAS, SEI, IET and CSI. He has been guiding research works in the area of fault tolerant computing, software safety and security, big data analytics and cognitive radio networks and intelligent systems.

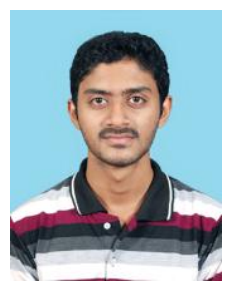

Arjun M. is doing computer science and engineering at Coimbatore Institute of Engineering and Technology affiliated to Anna University Chennai, India.

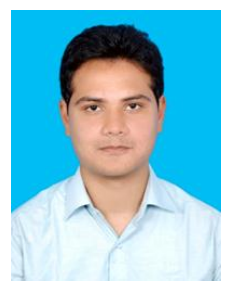

Sarathy T. J. Venkatesh is doing computer science and engineering at Karpagam College of Engineering affiliated to Anna University Chennai, India.

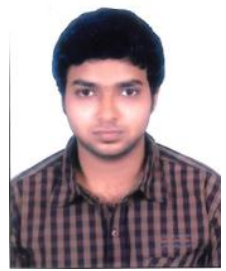

Veejay Adeesh is doing computer science and engineering at Karpagam College of Engineering affiliated to Anna University Chennai, India. 